\title{
Optoacoustic Evaluation of Endotracheal Tube Depth in Pediatrics
}

Technological advancement is a cornerstone in the practice of respiratory care. Applying new technology to solve clinical problems requires careful investigation to ensure the safety and efficacy of new devices, protocols, and treatment modalities. New technologies are often rapidly incorporated into daily clinical practice before high-quality data are available to guide clinical practice. Thus, new devices used in respiratory care need to be evaluated quickly after being introduced into the marketplace to validate their efficacy and safety before they are used extensively in patients.

Despite various proposed methods and technologies to guide endotracheal tube (ETT) depth in infants and children, ${ }^{1-5}$ ETTs are frequently incorrectly positioned in this patient population, although there is a large variance in the proportion reported in the literature. ${ }^{4,6}$ Chest radiography remains the standard of care for confirming appropriate ETT position within the trachea, which requires equipment and personnel to be brought to the bedside. Despite digital machines being widely available, there still may be a significant delay between ETT placement and chest imaging. For patients intubated outside the hospital, chest radiography cannot be performed until the patient arrives in a setting where this technology is available. Thus, there is great potential value in a device that can rapidly evaluate ETT position in real-time at the bedside.

In this issue of RESPIRATORY CARE, Volsko and colleagues investigated the use of a new optoacoustic device to evaluate ETT depth in pediatric subjects. ${ }^{7}$ In this pilot study, they demonstrated the utility of this new technology to rapidly (ie, within $45 \mathrm{~s}$ ) evaluate ETT depth in a cohort of 26 children. The procedure was relatively straightforward, with the acoustic detector placed at the sternal notch and a 1.0-mm optical fiber inserted through the ETT. Optical fibers were of a custom length for each ETT size. No adverse clinical events were noted. ETT position was de-

Dr Cheifetz discloses relationships with Philips, Up-to-Date, and the National Heart, Lung, and Blood Institute. Dr MacIntyre discloses relationships with Breathe Technology, InspiRx Pharma, and Ventec. The other authors have no conflicts to disclose.

Correspondence: Andrew G Miller RRT RRT-ACCS RRT-NPS, Duke University Medical Center, 2301 Erwin Road, P.O. Box 3911, Durham, North Carolina. E-mail: andrew.g.miller@duke.edu.

DOI: $10.4187 /$ respcare. 06687 tected accurately in 23 of the 26 subjects. Statistical analysis revealed a high level of agreement between the new device and chest radiography, as indicated by a correlation coefficient $\left(\mathrm{R}^{2}\right)$ of 0.91 and excellent agreement on the Bland-Altman analysis of bias and precision.

See the Original Study on Page 1463

This is an excellent example of a pilot study of new technology. However, the results need to be interpreted with caution. First, a convenience sample of 26 subjects from a single center is insufficient to adequately evaluate this technology. A larger, multi-center study is required to better account for heterogeneity in patient populations, provider capability, equipment and techniques, and other support systems. Second, despite generally accurate assessments, the difference between radiography and the optoacoustic device for 2 ETTs were $1 \mathrm{~cm}$ and $0.7 \mathrm{~cm}$, and a signal was undetected in a third subject. This means that the optoacoustic device provided a high level of accuracy for $88 \%$ of ETTs, which is similar to a simple approach evaluated by Phipps et $\mathrm{al}^{4}$ in which they found that $75 \%$ of ETTs were properly positioned on initial chest radiography using the internal diameter of the ETT $\times 3$ as the initial depth. Phipps et $\mathrm{al}^{4}$ also found that, when using the ETT size predicted by the PALS algorithm or Broselow chart (estimated weight based on patient length), the accuracy increased to $81 \%$ and $85 \%$, respectively. Third, no ETTs were at the carina or in a major bronchus, and thus the ability of the new device to detect this serious problem is not known. Finally, because none of the ETTs were evaluated in emergency or emergent situations, this device will require further investigation to evaluate its ability to detect incorrectly positioned ETTs in patients receiving cardiopulmonary resuscitation, as well as those outside the hospital setting.

Despite the limitations of the current study, this technology has real potential to improve airway management in pediatric patients. In particular, its utility in premature infants is of great interest because there are no currently available rules to accurately predict ETT depth in infants weighing $<1 \mathrm{~kg} .{ }^{8-10}$ Premature lungs are at particular risk of adverse events from incorrectly positioned ETTs, including pneumothorax, pulmonary interstitial emphysema, and endobronchial surfactant delivery. Evaluation of this 


\section{EDITORIALS}

device in premature and term neonates is well warranted, as is a multi-center trial in pediatric subjects enrolling a larger sample size. In addition, the utility in out-of-hospital airway management may also be of interest, given that up to $79 \%$ of pediatric patients intubated in the field arrive with the ETT placed too deep. ${ }^{11}$ Use of the device by transport teams and emergency medical services as well as during intrahospital transport may help reduce adverse events related to ETT migration.

\section{Andrew G Miller RRT RRT-ACCS RRT-NPS Kyle J Rehder MD Ira M Cheifetz MD FAARC Neil R MacIntyre MD FAARC Duke University Medical Center Durham, North Carolina}

\section{REFERENCES}

1. Chou EH, Dickman E, Tsou PY, et al. Ultrasonography for confirmation of endotracheal tube placement: a systematic review and meta-analysis. Resuscitation 2015;90:97-103.

2. Galicinao J, Bush AJ, Godambe SA. Use of bedside ultrasonography for endotracheal tube placement in pediatric patients: a feasibility study. Pediatrics 2007;120(6):1297-303.
3. Kattwinkel J, Perlman JM, Aziz K, et al. Neonatal resuscitation: 2010 American Heart Association guidelines for cardiopulmonary resuscitation and emergency cardiovascular care. Pediatrics 2010; 126(5):e1400-e1413.

4. Phipps LM, Thomas NJ, Gilmore RK, et al. Prospective assessment of guidelines for determining appropriate depth of endotracheal tube placement in children. Pediatr Crit Care Med 2005;6(5):519-22.

5. Sheth M, Jaeel P, Nguyen J. Ultrasonography for verification of endotracheal tube position in neonates and infants. Am J Perinatol 2017;34(7):627-32.

6. Volsko TA, McNinch NL, Prough DS, Bigham MT. Adherence to endotracheal tube depth guidelines and incidence of malposition in infants and children. Respir Care 2018;63(9):1111-17.

7. Volsko TA, Petrov Y, McNinch NL, Prough DS, Anderson CR, Bigham MT. Accuracy and precision of an optoacoustic prototype in determining endotracheal tube position in children. Respir Care 2018; 63(12):1463-1470.

8. Flinn AM, Travers CP, Laffan EE, O'Donnell CP. Estimating the endotracheal tube insertion depth in newborns using weight or gestation: a randomised trial. Neonatology 2015;107(3):167-72.

9. Leung C. Optimal insertion depth for endotracheal tubes in extremely low-birth-weight infants. Pediatr Crit Care Med 2018;19(4):328-31.

10. Peterson J, Johnson N, Deakins K, Wilson-Costello D, Jelovsek JE, Chatburn R. Accuracy of the 7-8-9 rule for endotracheal tube placement in the neonate. J Perinatol 2006;26(6):333-36.

11. Orf J, Thomas SH, Ahmed W, et al. Appropriateness of endotracheal tube size and insertion depth in children undergoing air medical transport. Pediatr Emerg Care 2000;16(5):321-27. 